\title{
LA GESTIÓN DE RIESGOS EN LA TOMA DE DECISIONES DE LOS AGENTES
}

\section{RESUMEN}

El presente documento tiene como objetivo presentar casos en los cuales los agentes involucrados enfrentaron situaciones en las cuales tomaron decisiones, las cuales no siempre fueron afortunadas. Asimismo, se presenta una breve discusión sobre la importancia de la gestión del riesgo, que debería ser utilizada como una herramienta para la toma de decisiones, antes que como otra de las decisiones que tomamos los agentes. Con este propósito se discute y analiza la definición de riesgo y conceptos relacionados

JEL: D81, G32, G11

PALABRAS CLAVE: Riesgo, gestión del riesgo, decisiones, estrategia

\begin{abstract}
This document has the objective of presenting some cases in which the agents related to them faced a decision-making situation, they made the decision, but its result was not necessarily a fortunate outcome. In order to understand these cases in the context of risk management (RM), this document discusses the importance of RM, not as another decision that people undertake, but as an important tool in the context of decision-making. This is why the document is focused on the discussion, analysis and understanding of the definition of risk and its related concepts
\end{abstract}

JEL: D81, G32, G11

KEYWORDS: Risk, risk management, decisions, strategy

\footnotetext{
A Edwin Navarro es Investigador Docente de la Escuela Profesional de Economía. Facultad de Ciencias Contables, Económicas y Financieras de la Universidad de San Martín de Porres (USMP). Lo expresado en el presente artículo es mi entera responsabilidad y no refleja, ni representa la posición de las entidades para las cuales trabajo o les doy asesoría.
} 


\section{INTRODUCCIÓN}

“..although people widely believe that they are inherently good decision makers, this belief is an illusion - a dangerous one." Carl Spetzler, Hannah Winter and Jennifer Meyer in Decision Quality

"Who is more valuable, the politician who avoids a war or the one who starts a new one (and is lucky enough to win)?...We glorify those who left their names in history books at the expense of those contributors about whom our books are silent. We humans are not just a superficial race (this may be curable to some extent); we are a very unfair one." Nicholas Taleb, in The Black Swan: The Impact of the Highly Improbable

"These pilots were not behaving like humans beings, like people who are experiencing this for the first time... Inmediately after the bird strike, they are turning back for the airport. Just as in the computer sims, correct?... They obviuosly knew the turn and exactly what heading to fly....No one has ever trained for an incident like that, no one.... I am not questioning the pilots. They are good pilots. But they've clearly been instructed to head for the airport immediately after the bird strike. You've allowed no time for analysis or decisión making. In these simulations, you've taken all the hummanity out of the cockpit." Captain Chesly Burnett "Sully" Sullenberger (pilot who landed US Airways Flight 1549 in the Hudson River)

El presente artículo tiene por objetivo mostrar porque la gestión de riesgos, lejos de ser un proceso complejo, burocrático y hasta tedioso, que se considera aislada de otras decisiones que tomamos, y que se vende cómo que debe ser gestionada sólo por expertos, es en realidad una actividad espontánea e interesante y que forma parte y está integrada a las diversas actividades de nuestro día a día.

Como se mostrará en el documento, no siempre nuestras decisiones son buenas, pero podríamos mejorarlas si entendiéramos mejor qué es el riesgo, de allí que la motivación es hacer que quién lea éste documento se sienta cómodo para entender (i) qué es riesgo, (ii) y como ese conocimiento puede ser utilizado para gestionar riesgos y eso nos pueda ayudar a tomar mejores decisiones; es decir, ser mejores "gestores del riesgo".
El documento destaca la filosofía que plantean Nicholas Taleb, Grant Purdy, y Alex Sidorenko ${ }^{1}$, quienes enfatizan que siempre no sabremos qué ocurrirá en el futuro, pero no por eso deberíamos abstenernos de tomar riesgos, puesto que las decisiones que tomamos las hacemos para lograr algún objetivo, y de hecho siempre los objetivos que perseguimos estarán sujetos al riesgo de que no se puedan cumplir, tal como lo planificamos

El documento se desarrolla en tres secciones. En la primera, se presentan varias breves reseñas de situaciones del pasado en las cuales diversos agentes se encontraron en situaciones en las que tuvieron que tomar decisiones, las cuales no siempre fueron afortunadas. Luego, en la segunda parte se discute sobre lo que se consideran son las más importantes decisiones que toman los agentes. En la tercera, se discute sobre la definición del riesgo y sobre otros conceptos que están detrás del riesgo. En la última parte se presenta y se discute la conclusión.

\section{LA GESTIÓN DE RIESGO EN ALGUNAS HISTORIAS $Y$ SITUACIONES DEL PASADO}

Primero que nada quiero enfatizar que el riesgo tiene que ver con el futuro y no con el pasado. Una de las industrias que a mí me gusta estudiar es la industria de la aeronáutica porque debe ser una de las pocas industrias que estudian sus "fracasos" para mejorar. En ese sentido, el pasado podría servirnos para entender mejor el riesgo, especialmente cuando se estudian situaciones con las cuales podríamos entender y explicar mejor cómo es que se debió haber gestionado riesgos en la toma de decisiones. Debo aclarar que cuando me refiero a gestionar riesgos, esto de por si no implica que el resultado de la situación presentada haya terminado con una consecuencia favorable ${ }^{2}$.

Como se verá al final de documento, en una tabla se resume y busca identificar cómo en los casos presentados a continuación se podría aplicar los conceptos discutidos en el presente documento.

\footnotetext{
1 Debo reconocer que el principal motivador del presente artículo fue leer (escuchar en realidad) una entrevista que le hizo Alex Sidorenko a Grant Purdy. Grant Purdy podría considerarse como una leyenda en la gestión de riegos, ya que es uno de los autores del estándar Australiano Neo-Zelandés para la gestios de riesgo. Este estándar es el precursor de lo que hoy conoce como la ISO-31000.

2 Mucho se critica respecto a analizar situaciones del pasado para cuestionar decisiones que se tomaron en el pasado, al punto que se utiliza el término "todos somos profetas del pasado". Uno de los grandes mitos, para quienes sabemos algo pasado". Uno de los grandes mitos, para quienes sabemos algo te asegura que siempre serás un ganador o que tendrás que asumir pérdidas para lograr un objetivo.
} 


\section{Caso 1: E1 futbolista que estuvo a punto de perderse un mundial.}

La primera situación es un caso reciente (del año 2018) de un jugador de la selección peruana quien estuvo a punto de no jugar en el mundial de Rusia de 2018. Según la información que se tiene, este jugador fue suspendido porque se le había encontrado una sustancia prohibida, después de habérsele efectuado el examen de dopaje, el cuál es obligatorio para a un determinado jugador elegido por sorteo.

Según argumentos del futbolista y de información propalada en los medios de comunicación escrita, el futbolista estima que fue contaminado cuando bebió un mate de anís, el mismo que al parecer se le sirvió en una jarra que bien pudo haber estado contaminada con residuos de un mate de coca. De acuerdo al jugador, bebió de la jarra con toda confianza porque asumió que la zona donde tomó el mate de anís estaba dentro de la zona de seguridad. Después, en la investigación, él mismo descubrió que esa zona no estaba dentro de la zona de seguridad ${ }^{3}$.

\section{Caso 2: Catástrofe aérea: El vuelo 603 de Aeroperú.}

Minutos después de iniciado el día 02 de octubre de 1996, decoló desde el Aeropuerto Jorge Chávez del Callao (Perú) un Boeing 757, de la extinta aerolínea Aeroperú, con destino al Aeropuerto Arturo Merino de Santiago de Chile. El avión, antes de decolar, había sido objeto de una limpieza y mantenimiento, para lo cual, quienes se encargaron de la limpieza exterior del avión (lavado con agua presurizada), habían tenido el cuidado de "sellar" los puertos estáticos ("static ports") y "pitots" con cinta adhesiva ("masking tape") para que el agua no se introduzca dentro de tales partes del avión.

Terminado el proceso de limpieza, los trabajadores encargados olvidaron retirar los "sellos" (lo cual se demostró en la investigación posterior del accidente, luego del rescate de los escombros en el mar). Lo que resulta más sorprendente aún es que, ni los supervisores, ni la tripulación de la aeronave hayan efectuado una verificación para asegurarse de que, tanto los puertos estáticos como los "pitots", hayan estado libres de los "sellos", antes de proceder con el vuelo.

\footnotetext{
3 En las concentraciones de deportistas se considera la "zona de seguridad" a aquellos ambientes en los cuales se aplican controles para que un deportista no se contamine, especialmente cuando ingiere sus alimentos, incluyendo las bebidas que toma, de cualquier tipo.
}

Como era de esperarse, dado que los puertos estáticos y los "pitots" cumplen funciones determinantes para proveer información a los sistemas de aeronavegación de los aviones, los pilotos del vuelo 603 estaban "ciegos". Tras volar por varios minutos, sin tener idea de a qué velocidad y a qué altitud volaba la aeronave, además de no tener visibilidad por ser de noche, el avión se precipitó en el Océano Pacifico, a pocas millas del Aeropuerto Jorge Chávez.

Se pudo haber especulado y encontrado evidencia de negligencia de porque los trabajadores se olvidaron de retirar los "sellos", pero una causa próxima de porque se olvidó dicho retiro es el hecho de que muchas veces como personas tomamos decisiones por apuro, ya sea porque nos obligan a terminar alguna tarea, o porque estamos retrasados y tenemos que culminar un encargo urgente o porque le "queremos ganar al tiempo", o peor, nos encontramos en una situación crítica. En este tipo de situaciones, las decisiones que tomamos tienen alta probabilidad de tener consecuencias fatales, como fue el caso del avión siniestrado. Es muy probable que la decisión de la tripulación fue partir porque estaban contra el tiempo y asumieron que todo estaba en orden.

\section{Caso 3: De potencial postulante de} Google a multimillonario: Elon Musk.

Elon Musk confesó en una entrevista que cuando había terminado la universidad y había evaluado varias alternativas para buscar trabajo, la opción más interesante le resultaba postular a una naciente empresa llamada Google. Sin embargo, ya estando en la cola para presentar sus credenciales competitivas (léase, $\mathrm{CV}$ ), prefirió salirse de la cola, visualizando que en el futuro él podría estar al otro lado; es decir, contratando personal para sus empresas.

Elon Musk es uno de los personajes a quién admiro porque no sólo le gusta tomar riesgos, sino porque también sabe gestionarlos, porque le gusta entender la complejidad para tomar sus decisiones, y porque tiene una alta tolerancia a la pérdida, al punto de estar dispuesto a perderlo todo. Esto ha ocurrido en los diversos emprendimientos que él ha llevado a cabo en

\footnotetext{
4 El trabajador responsable retirar los sellos finalmente fue procesado y condenado penalmente por negligencia. El título de artículo sobre el cual se basa esta historia es sugestivo: "MASKING TAPE BLINDFOLDS 757-PITOT BLOCKED (Lima 1996) CRASHES AND MIRACLE LANDINGS- Sixty Narratives (How, When ... And Most Importantly Why), de Christopher Bartlett. Ver Referencias.
} 
su vida, incluso si se considera que lo dejó todo en su nativa Sudáfrica, para primero mudarse a Canadá y luego a los Estados Unidos. Esto se confirma en un artículo publicado por la revista The Economist, que dice: “(el emprendimiento) de Musk no sólo se sustenta en ideas y su encanto (como persona). Otros dos atributos resaltan (en su personalidad), que son las formas de cómo Musk aborda el riesgo y (cómo) gestiona la complejidad". 5

Musk, después de estudiar en la universidad, física y economía, se mudó a California para hacer un postgrado en física en la Universidad de Stanford, pero a los pocos días de estar allí, abandonó sus estudios de postgrado para formar una empresa de software con su hermano, denominada Zip2, en los días en que el uso de la internet y el software de visualización de códigos postales estaban en su infancia. Debido a las bondades de su software, Compaq compró su empresa en 1 999. Según el mismo artículo del The Economist, la transacción fue por USD 341 millones (obviamente no todo era suyo).

Musk lejos de retirarse y vivir de sus ganancias, el total recibido lo invirtió en la creación de una empresa denominada X.com, una compañía cuyo propósito era competir con los bancos, en la gestión de servicios bancarios. No fue tan exitoso, pero a su emprendimiento le dio un giro y sólo se dedicó a proveer servicios transaccionales para competir frontalmente con los bancos, lo cual si fue exitoso. X.com luego se "convirtió" en PayPal, y en un momento determinado fue adquirida por eBay por USD 1,500 millones.

Igual que antes, cualquier persona con las ganancias obtenidas probablemente habría optado por retirarse. A estas alturas Musk ya era un multimillonario. Sin embargo, Musk no es cualquier persona, y todas sus ganancias las invirtió en otros dos emprendimientos: Tesla y Space X. Como dice el artículo del The Economist, “(Musk) cada vez toma más riesgos, y más grandes" ". Con Tesla y Space X estuvo a punto de quebrar por grandes problemas de liquidez, cuando se desencadenó la crisis financiera de 2008. Incluso, Larry Page, fundador de Google, tenía listo el cheque para efectuar el aporte de capital y sacar de la bancarrota a las empresas de Musk. Por fortuna, Musk firmó un contrato

\footnotetext{
5 "How Elon Musk does it: The Falcon Heavy"s Creator is trying to change more worlds tan one-Faliure is most definitively an option.” The Economist, February, 10th., 2018.

6 Ver The Economist, Idem.
}

con el Estado Americano para la provisión de cohetes para la NASA. Eso lo salvó ${ }^{7}$.

\section{Caso 4: E1 Presidente versus el General: ¿la posible tercera guerra mundial?}

Una historia fascinante al finalizar la segunda guerra mundial es el caso de insubordinación por parte del General Mac Arthur contra el Presidente Harry Truman ${ }^{8}$. Como se sabe, después de que Estados Unidos lanzara sus bombas atómicas sobre Hiroshima y Nagasaki, a Japón no le quedo más que rendirse. Para el que no lo sabe, el General Mac Arthur fue quien firmó, en representación de los Estados Unidos, la rendición de Japón. Mac Arthur se convirtió en un héroe nacional, pero no sólo eso, sino que cuando se le asignó el trabajo de "reconstruir" Japón, ganó poder, al punto de que el Emperador de Japón de una forma u otra se subordinó a sus órdenes, aunque se cuenta que Mac Arthur siempre fue respetuoso del Emperador.

A Mac Arthur también se le asignó la labor de dirigir las operaciones en el conflicto en el cual Corea del Norte, con el apoyo de los rusos y chinos, buscaba tomar el control de toda Corea. Estados Unidos, utilizando su poder bélico prácticamente desapareció del mapa la infraestructura física de Corea del Norte, pero inmediatamente la China de Mao Tse Tung tomó el liderazgo para defender a Corea del Norte, con lo cual el asunto se complicó para los Estados Unidos. Para Mac Arthur el asunto era simple de resolver porque consideraba que Estados Unidos tenía el suficiente poder para vencer a los chinos, incluso, según Mac Arthur, se podía utilizar todo el poderío nuclear para este fin. Sin embargo, para tomar acciones requería la autorización del Comandante en Jefe; es decir, el Presidente Truman. Pero como Truman no le dio la autorización, Mac Arthur hábilmente comenzó a utilizar su poder y popularidad, buscando convencer tanto a los Representantes del Congreso Americano y al pueblo Norteamericano para presionar a Truman.

Truman que había sido el vicepresidente de Roosevelt había sido advertido por este último, tildando a Mac Arthur como una persona muy peligrosa. Lo que Truman estaba experimentando

7 La biografia de Musk y la historia completa de sus emprendimientos está completa en el libro de Ashlee Vance, "Elon Musk: Tesla, Sapce X and the Quest for a Fantastic Future," (2015)

8 Un libro interesante sobre este caso es el escrito por H.W. Brands, "The General vs. the President: Mac Arthur and Truman at the Brink of Nuclear War.”(2016) 
con Mac Arthur en esos momentos era que la advertencia era válida. Truman optó por licenciar de su cargo a Mac Arthur.

Pero lo más interesante de esta historia es que Mac Arthur volvió a Estados Unidos para mostrarse ante la opinión pública de los Estados Unidos de que Truman lo había maltratado y que la incompetencia de Truman perjudicaría en el largo plazo el rumbo de la guerra fría, en contra de los Estados Unidos. Posteriormente, cuando el Congreso investigó, Mac Arthur quedó mal parado y su poder y popularidad se fueron desvaneciendo. Hoy podríamos afirmar que Truman tomó una buena decisión, especialmente porque contaba con información incompleta del poder bélico de los rusos, que de seguro hubieran apoyado a los chinos, con lo cual el mundo posiblemente habría entrado a una tercera guerra mundial.

\section{LAS DECISIONES (FINANCIERAS $Y$ OTRAS) QUE TOMAN LOS DIVERSOS AGENTES}

Zvi Bodie y Robert Merton, en su libro Finanzas, identifican y resumen las principales decisiones financieras que toman las empresas y las familias. Según ambos autores, las familias afrontan cuatro tipos principales de decisiones financieras: (i) de consumo y de ahorro, (ii) de inversión, (iii) de financiamiento, y (iv) de administración del riesgo. De otro lado, las empresas, básicamente estarían concentradas en las siguientes decisiones financieras: (i) la planeación estratégica, (ii) la elaboración del presupuesto de capital, (iii) la forma como financiaran sus activos (estructura de capital), y (iv) la gestión del capital de trabajo9.

El primer comentario respecto a la propuesta de Bodie y Merton es que no sólo las familias y las empresas toman decisiones financieras, sino que también otros tipos de agentes. Dentro de los otros agentes se puede considerar a los Estados (a través de los gobiernos), los organismos multilaterales (como el Fondo Monetario, el Banco Mundial o la ONU), los organismos no gubernamentales (ONGs), los gobiernos locales, y hasta las iglesias.

El segundo comentario es que si bien importan mucho las decisiones financieras, muchas otras decisiones trascienden a lo exclusivamente financiero, aunque en realidad casi todo lo que

9 Finanzas, Zvi Bodie y Robert C. Merton, Prentice Hall, Mexico, 1999, págs. 4-7. hacemos los homo sapiens está vinculado, de una forma u otra, a las finanzas. De hecho, debe considerarse que cualquier entidad que esté administrada por personas "tomará" decisiones.

El tercer comentario es que nos es correcto encasillar los tipos de decisiones para un solo tipo de agente. Es un error pensar por ejemplo que sólo las empresas efectúan el planeamiento estratégico. No es difícil pensar en la cantidad de decisiones estratégicas que tomamos las personas a largo de nuestra existencia. Igual, las empresas toman decisiones de consumo y de ahorro, al igual que los Estados, lo organismos multilaterales o las ONGs.

El cuarto comentario, y el que más me interesa destacar para el presente documento, es que la decisión de administración de riesgo ${ }^{10}$ que plantean Bodie y Merton, en sí no debería considerarse como una decisión, sino como una herramienta adicional para mejorar nuestra toma de decisiones. Considero que esto es así porque cuando tomamos alguna decisión, por definición deberíamos estar tomando algún riesgo. Lo que acabo de plantear está alineado con Hubbart, quién plantea que

[A]sí como la gestión de riesgo debería ser un subconjunto de la administración de una organización, el análisis del riesgo debería ser un subconjunto del análisis de decisión. Las decisiones no pueden estar basadas enteramente en el análisis de riesgo, sino que requieren un análisis de los potenciales beneficios si los gerentes deciden aceptar un riesgo ${ }^{11}$.

Además de lo anterior, más interesante aún es considerar como nosotros como agentes estamos expuestos y operamos en sistemas complejos, con lo cual se hace más importante el uso de herramientas para la mejor toma de decisiones. Por ejemplo, no es difícil pensar en situaciones en las cuales otros agentes toman algunas decisiones para lograr sus objetivos, pero dichos objetivos no necesariamente están alineados con nuestros objetivos y viceversa. Esto es de esperarse porque en general operamos en un mundo en el cual los sistemas interactúan, muchas de las veces de manera caótica y compleja.

10 En lo que sigue del documento el término "administración de riesgo" será usado de manera indistinta también como "gestión del riesgo"

11 The Failure of Risk Management: Why It's Broken and How to Fix It, Douglas W. Hubbard, John Wiley \& Sons, Inc, 2009, pág. 92. (Traducción propia). 
Es imposible pensar hoy que vivimos en sistemas simples, en los cuales sería simple predecir el futuro. Como agentes ya no participamos, o lo hacemos con menos frecuencia, en sistemas en los cuales se puede aplicar el análisis determinista. Por el contrario, cada vez en más común participar en sistemas complejos y caóticos, por lo que el análisis ya no aplica. En sistemas simples sería más fácil efectuar predicciones y proyecciones porque en ellos se pueden identificar patrones de comportamiento, pero en los caóticos los patrones no son fácilmente identificables y además estos patrones son aleatorios. De hecho en los segundos, no existen reglas establecidas que se tienen que cumplir. Los primeros sistemas son lineales, los otros definitivamente no lo son.

Taleb justamente es una de las personas que más crítica a la "academia" porque sus teorías siempre están sustentadas en asumir que los sistemas en los cuales operamos son lineales. Un asunto interesante a tomar en cuenta es que los sistemas predecibles podrían evolucionar a sistemas caóticos, si por ejemplo algún o varios factores de un sistema determinístico cambian y se vuelven aleatorios, lo cual los lleva a tener un comportamiento no predecible; es decir, caótico.

Taleb está convencido de que el mundo en el cual interactuamos los humanos es uno del tipo caótico y que las anormalidades son más frecuente de lo que pensamos, al punto de plantear de que lo normal para nosotros debería ser como ubicarnos en los extremos de una curva normal (como en la campana de Gauss). De allí que Taleb argumenta que es más importante siempre pensar en eventos extremos que pensar en eventos "normales", a los cuales siempre tenemos la tendencia a pensar que seguirán ocurriendo Por eso, Taleb considera que conceptos como la media y la desviación estándar resultan irrelevantes ${ }^{12}$.

Los típicos ejemplos de sistemas caóticos son por ejemplo la ruta que siguen los huracanes y tifones en varias partes del planeta. Es imposible determinar con precisión la ruta que seguirán. En economía, es imposible predecir con exactitud que la economía crecerá a una determinada tasa de crecimiento en un período de tiempo determinado. En los sistemas caóticos no hay una sola solución o una sola respuesta. Podrían ser muchas, pero aun así, eso no es predecible al $100 \%$. Siempre habrá un margen de error.

\footnotetext{
12 Toda la filosofia de Taleb se basa en este principio, la cual está expuesta en todos sus libros que tratan sobre la incertidumbre. A esa colección de libros Taleb le ha puesto la etiqueta de "Incerto".
}

Quizá por lo planteado líneas arriba, para nosotros sea cada vez más difícil tomar decisiones porque ya no sólo basta mirar el pasado para hacer una predicción de nuestros objetivos, sino que también nuestros objetivos ya no pueden ser fijos y pueden cambiar en el tiempo y además de manera muy rápida, por lo que nuestras decisiones también se verán afectadas. Necesitamos mucho más que asumir que lo que pasó antes volverá ocurrir en el futuro; es decir, pensar como Taleb, que la "normalidad" ahora es otra.

Por lo anterior, considero que una buena forma de ayudarnos para mejorar nuestras decisiones es entender la teoría de los fractales, propuesta por Benoit Madelbrot. Para entender qué es un fractal podríamos utilizar a un brócoli. Cuando observamos un brócoli de lejos, veremos que el brócoli tiene un patrón de ramas, pero si lo observamos a nivel de cada rama, nos daremos cuenta que cada rama tiene el mismo patrón del brócoli original, y si lo investigamos más de cerca, notaremos que las ramas de la rama tendrán el mismo patrón del brócoli. Un fractal es un sistema que tiene dimensiones fraccionadas dentro del mismo. Es una fracción de una dimensión. La dimensión sería el brócoli y cada rama del brócoli es en sí un brócoli, pero de dimensiones menores dentro de una dimensión mayor que es el brócoli. Cómo los sistemas complejos son caóticos, la teoría de los fractales nos podría ayudar a entender mejor la complejidad y por tanto, ayudarnos para tomar mejores decisiones.

De otro lado, en el libro Decision Quality, sus autores proponen que para tomar mejores decisiones en principio deberíamos mejorar nuestras habilidades para la toma de decisiones "porque las decisiones [que tomamos] son tan importantes para nuestro presente y futuro, por lo que hacerlas bien debería ser una prioridad. Y por supuesto, tomar buenas decisiones es algo que podemos aprender a hacer". Como también lo apuntan sus autores, incluso gente preparada para dirigir y tomar decisiones reciben poco o ningún entrenamiento para la toma decisiones ${ }^{13}$. Peor aún, si quienes toman decisiones consideran que operan en sistemas simples.

En el mismo libro de Decision Quality, se argumenta que la calidad de nuestras decisiones dependerá de los siguientes elementos: (i) un marco [de referencia] apropiado, (ii) alternativas creativas, (iii) información importante y

13 Decision Quality: Value Creation from Better Decisions, Spetzler, Carl, Winter Hannah, and Meyer Jennifer, Published by John Wiley \& Sons, Inc., Hoboken, New Jersey, Pág. 5. 
confiable, (iv) valores claros y su balance, (v) razonamiento sólido, y (vi) actitud precisa para actuar ${ }^{14}$. Todos están encadenados, sin embargo, enfatizan en lo siguiente: "tres asuntos deben ser aclarados: las alternativas definen qué podemos hacer, la información captura qué sabemos y creemos (pero que no podemos controlar) y los valores representan qué [objetivos] deseamos y esperamos lograr. Juntos estos tres elementos forman la base de nuestras decisiones. Ellas deben combinarse usando el razonamiento que nos guía a la mejor elección de lo que deseamos (valores) y a la luz de lo que sabemos (información). El razonamiento nos ayuda a entender qué deberíamos hacer, creando claridad en [nuestras] intenciones. Sin embargo, una intención tiene poco valor práctico. Para tomar una decisión real, debemos actuar. Por ello, la actitud para actuar debe ser una parte integral de [una] decisión."15

Tomando en cuenta lo anterior, es por eso que siempre he considerado que casi todas las decisiones que tomamos son del tipo estratégico y las que no lo son dependen de un objetivo estratégico, algo así como que las decisiones tienen un objetivo y final, pero también tienen objetivos intermedios, pero que dependen del principal. Para entender mi posición consideremos que se entiende por estrategia. Dos libros a los que he tenido acceso tratan sobre el concepto de la estrategia: el primero es el de Lawrence Feedman y el otro es de Esteban Hnyilicza.

Respecto al concepto de estrategia, Freedman siempre destaca que la estrategia debe ser entendida como un proceso que va evolucionando, que no es estático y que nos obliga a ir cambiando nuestra forma de actuar, de acuerdo a como se va presentando el entorno en el cual operamos ${ }^{16}$. Por tanto, esto implicaría que las nuestras decisiones no tendrían que ser estáticas y por el contrario deberían irse ajustando. Esto de por sí le añade complejidad a nuestro proceso de toma de decisiones.

En cuanto a Hnyilicza, su definición de estrategia debería ser considerada como la mejor definición del concepto. De hecho está muy alineada a lo que plantean los autores del libro Decisión Quality, pero además considero que nos puede ayudar a entender mejor la definición de riesgo, que es la principal motivación del presente documento. Según Hnylicza,

14 Idem, pág. 11

15 Idem, pág. 11

16 Strategy: A History, Freedman, Lawrence, Oxford University Press, 2016.
En términos generales, una estrategia es una concepción instrumental con el poder de traducir una visión sobre el futuro a acciones concretas que hacen posible articular su proyección sobre la realidad. La estrategia es lo que enlaza la visión con la acción ${ }^{17}$.

Visto desde esta perspectiva, entonces el objetivo sería la realidad que buscamos y las acciones concretas serían el conjunto de decisiones que tomamos para conseguir un determinado objetivo. Lo interesante es que los objetivos no sólo deberían estar sujetos a riesgos, sino que también las acciones que nos llevarían al logro de un determinado objetivo porque las acciones también tienen objetivos intermedios, los cuales nos deberían conducir al logro del objetivo final.

\section{EL RIESGO Y SU VINCULACIÓN CON LAS DECISIONES DE LOS DIVERSOS AGENTES}

Contrariamente a lo que se piensa, los expertos en riesgos parecería que todavía no se ponen de acuerdo sobre cuál debería ser la correcta definición de riesgo. Esto de por sí genera gran confusión. Cada experto en riegos o cada estándar que trata sobre la gestión riesgos consideran que su definición debería ser considerada como la correcta $^{18}$.

De las varias definiciones que he estudiado, considero que las mejores son las siguientes:

Bodie y Merton:

La incertidumbre existe siempre que no se sabe con seguridad lo que ocurrirá en el futuro. El riesgo es la incertidumbre que importa (sic) porque incide en el bienestar de la gente. Así pues, la incertidumbre es una condición necesaria pero no suficiente del riesgo. Toda situación riesgosa es incierta, pero puede haber incertidumbre sin riesgo. ${ }^{19}$

\section{Hubbart:}

Incertidumbre versus riesgo y las medidas de cada uno.

- Incertidumbre. La falta de completa certidumbre- esto es, la existencia de

17 Hnyilicza, Esteban, El Abrazo Invisible: Hacia las Reformas de Tercera Generación, Fondo Editorial de la Universidad San Martin de Porras, 2005, Pág. 45.

18 Grant Purdy estima que en los diferentes estándares de gestión de riesgos se podrian encontrar hasta 40 formas diferentes de definirlo, pero Siderenko considera que podrian ser alrededor de 70.

19 Bodie y Merton, pág. 216. 
más de una posibilidad. No se conoce el "verdadero" resultado/estado/ consecuencia/valor.

- Medida de incertidumbre. Un conjunto de probabilidades asignadas a un conjunto de posibilidades. Por ejemplo, 'hay una posibilidad de $60 \%$ de que lloverá mañana, y una posibilidad de $40 \%$ de que no lloverá.'

- Riesgo. Un estado de incertidumbre donde algunas de las posibilidades involucra una pérdida, una lesión, una catástrofe, u otro resultado no deseado ([es decir,] ocurra algo malo).

- Medida de riesgo: Un conjunto de posibilidades cada una con probabilidades cuantificadas y pérdidas cuantificadas. Por ejemplo, '[en una empresa petrolera] Consideramos que hay una posibilidad de que el pozo petrolero estará seco con una pérdida de \$12 millones en los costos de perforación exploratoria. ${ }^{20}$

\section{ISO 31000:}

Efecto de la incertidumbre en los objetivos.

Nota 1: Un efecto es una desviación respecto a lo esperado. Puede ser positivo o negativo o ambos, y puede llevar, crear o resultar en oportunidades y amenazas.

Nota 2: Objetivos pueden tener diferentes aspectos y categorías, y se pueden aplicar a diferentes niveles.

Nota 3: Riesgo siempre se expresa en términos de las fuentes de riesgo, potenciales eventos, sus consecuencias y la posibilidad de que ocurran. ${ }^{21}$

Por muchos años he utilizado la definición de Zvi Bodie y Merton como la correcta y me ha servido para argumentar mis discusiones sobre el riesgo. La definición de Hubbart también me ha ayudado mucho para sustentar cuando me he enfrentado en discusiones sobre la diferencia entre incertidumbre y riesgo, y especialmente cuando se discute la forma cómo se mide uno $u$ otro concepto.

20 Hubbard, idem, pág. 80. (Traducción propia).

21 ISO 31000:2018 Risk management - Guidelines, Second Edition 2018-02, pág. 1. (Traducción propia)
Si bien ambas definiciones son muy interesantes, considerando la definición de la ISO 31000 (2018), podríamos deducir que ambas no estarían completas. Quizá por ello que considero que la mejor definición vigente debería ser la de la ISO 31000 (2018), por su alcance y fundamentación.

Si uno sólo lee la definición de la ISO 31000 (2018), sin sus notas, podría no ser fácil entender la definición de riesgo, pero analizando las notas aclaratorias (notas 1, 2 y 3 ) de los términos, efecto, objetivos y riesgo, se entenderá porque es la mejor definición. Además, considero que para completar el concepto del ISO 31000 (2018), deberíamos analizar otros conceptos importantes como son los de amenaza, vulnerabilidad, apetito, tolerancia y capacidad.

Lo que deseo mostrar es que para que exista el riesgo siempre deberán estar presentes los siguientes componentes: objetivo, consecuencia $\mathrm{o}$ impacto, evento, factor causal o causa $\mathrm{u}$ origen, y que el entendimiento del riesgo definitivamente nos debería servir para utilizar la gestión de riesgos como una herramienta que nos debería ayudar a tomar mejores decisiones.

Para entender la definición de la ISO-31000 (2018), yo podría empezar visualizando la siguiente secuencia: (i) un factor de riesgo (o causa de un evento) desencadena un evento, (ii) al ocurrir el evento, (iii) esto llevará a que se presenten consecuencias o impactos. Y la pregunta sería ¿y que afectan los impactos? La respuesta es que los impactos o las consecuencias son las que afectan a un objetivo. Con esto quedaría completa la definición de riesgo.

Con lo anterior entonces podríamos decir que para que exista riesgo, es necesario que (i) exista el objetivo o los objetivos que serán afectados, (ii) por las consecuencias o impactos, ante la (iii) ocurrencia de un evento, que se (iv) origina por la activación de uno o varios factores de riesgo. Como el factor de riesgo, el evento y los impactos están en el futuro y no han ocurrido (podrían ocurrir o no), son algo potencial y constituyen incertidumbre pura, entonces la definición de la ISO 31000 (2018) es correcta: "Es el efecto de la incertidumbre en los objetivos".

Pudiéramos definir cada uno de los elementos que componen el riesgo, con excepción del objetivo, lo cual ya fue discutido en la sección anterior del presente documento. En cada caso, las definiciones o son las que utiliza la ISO 31000 (2018) o reflejan mi entendimiento. 
El factor de riesgo refiere a un elemento que, por sí solo o en combinación con otros factores tiene el potencial para dar origen a un evento. Un evento es la ocurrencia o el cambio de un conjunto particular de circunstancias. Un evento puede tener una o más ocurrencias, y resultar de diversas fuentes y tener varias consecuencias o impactos. Un evento de por si es algo potencial porque todavía no ha ocurrido dado que se refiere al futuro y es una circunstancia esperada que no necesariamente ocurrirá o una circunstancia no esperada que ocurrirá. Una consecuencia o impacto es el resultado de la ocurrencia de un evento que afecta el logro de un objetivo, y que, dependiendo del contexto, podría tener efectos en cadena y dar lugar a otros eventos; es decir, podría ser el factor de riesgo de otros eventos. A esto último normalmente se conoce como la "propagación del riesgo".

Otros conceptos que merecen ser establecidos para entender mucho mejor el concepto de riesgo son los conceptos de amenaza, vulnerabilidad, nivel de riesgo, apetito al riesgo, tolerancia al riesgo y capacidad de riesgo.

Una amenaza es cualquier elemento que tiene el potencial de afectar, incluso de manera espontánea a otro elemento, con la intención o no, de manera directa o indirecta. Normalmente las amenazas son parte inherente de los factores causales que son los que originan un evento. La vulnerabilidad se refiere a las propiedades intrínsecas de un elemento que lo hacen susceptible de ser afectado por una o varias amenazas. Ambos conceptos son importantes porque el riesgo estará relacionada directamente con la amenaza y la vulnerabilidad. Debería esperarse que un evento tenga un mayor impacto cuanto más vulnerable sea un elemento frente una amenaza.

Lo anterior es importante para explicar el concepto del nivel de riesgo porque el efecto de la incertidumbre sobre el objetivo, léase riesgo, requiere ser estimado. La razón es, si no sé efectúa una estimación del riesgo, entonces no se contaría con un elemento para poder gestionarlo. Entonces debe entenderse por nivel de riesgo a la magnitud del efecto expresado en términos de la combinación de la probabilidad de que un factor causal o factores causales originen un evento y que ocurrido el evento, éste tenga consecuencias (impacto).

Como al final el nivel de riesgo mide el efecto sobre el objetivo, entonces una vez determinado este nivel deberá uno preguntarse si el efecto en realidad es algo esperado. Esto es bien importante porque yo entiendo que aquí entra a tallar el aspecto positivo del riesgo. Me explico. Supóngase que ocurre un evento y el efecto sobre el objetivo podría estar por debajo de lo que uno espera, entonces tendría sentido tomar más riesgo o incrementar la exposición al riesgo.

Para explicar mejor lo anterior podría incorporarse los conceptos de apetito, tolerancia y capacidad de riesgo. Debe entenderse como apetito al nivel de riesgo deseado (efecto esperado), nivel de riesgo que uno está dispuesto a tomar para el logro un objetivo. Pero podría ocurrir que el nivel de riesgo esté por encima del nivel deseado, pero que aun así uno esté dispuesto a tomar el riesgo. Esto último se conoce como tolerancia. Ahora imaginemos que uno quisiera tomar el riesgo para el logro de un objetivo, pero el nivel de riesgo sobrepasa lo que lo uno podría tolerar, entonces esto se conoce como capacidad de riesgo, que es el nivel máximo de riesgo que uno puede soportar, el cual, si excede, lo llevaría a uno a una situación en la cual su existencia se vería seriamente amenazada en extremo.

Considero que este es el momento oportuno para considerar que lo discutido en el presente documento respalda mi idea de porque la gestión de riesgo es verdaderamente una herramienta que nos ayuda a tomar mejores decisiones. Atando conceptos yo podría afirmar que los agentes toman decisiones en el tiempo para conseguir un determinado objetivo, que puede ser intermedio o final, pero que estos objetivos podrían estar plagados de incertidumbres que afectan dichos objetivos, pero esto implica que por ello no estemos dispuestos a correr riesgos. Considero que al final todo dependerá de qué nivel de riesgo estamos dispuestos a tolerar. Algo así como ¿cuánto estoy dispuesto a perder como perdida máxima, cuando "apuesto" por lograr un objetivo?

La mayoría de los conceptos discutidos en esta parte se han adecuado a los casos presentados en la primera parte del documento. Sugiero analizarlos. Esto se presenta en la Tabla 1.

\section{CONCLUSIONES}

Considero que después de la Segunda Guerra Mundial el concepto de riesgo ha ido evolucionado tremendamente, pero quienes lo han estudiado no han podido establecer las condiciones para tomar ventaja para ayudarnos a 
poder tomar mejores decisiones. Por el contrario, como lo plantea Grant Purdy nos hemos ido complicando, al punto que no existe una definición aceptable que sea el estándar o como lo plantea Nassin Taleb, quién considera que la "Academia" ha creado modelos para gestionar riesgo con supuestos lineales cuando la realidad es que en riesgo no existe la linealidad.

Peor aún, como lo plantean también Taleb y Purdy se ha creado una industria en la cual se vende la gestión de riesgo como una panacea, lo cual ha llevado no sólo a que no hayamos tenido una definición aceptable de riesgo, sino que tengamos un sinnúmero de estándares que se utilizan para cada tipo de riesgo. Considero que la ISO 31000 (2018) ha contribuido de manera importante a la definición del concepto, pero al igual que otros estándares muestra el sesgo para burocratizar la gestión del riesgo.
Los estándares de gestión de riesgo y los "gurús" de la gestión del riesgo han creado una confusión y han burocratizado la gestión del riesgo al punto de que un gran número de empresas cuentan con personal especializado, quizá no porque lo consideran adecuado para ayudarles a tomar mejores decisiones, sino porque la regulación lo exige o porque quién sabe es una moda impuesta por los sistemas de gestión que utilizan las empresas.

El propósito del presente documento principalmente se ha enfocado en mostrar que la gestión de riesgos debería servir como una herramienta para ayudarnos a tomar mejores decisiones. Considero que para eso debería servir la gestión de riesgo. 
Tabla 1

Conceptos relacionados a riesgo, aplicados a los casos presentados en la primera parte

\begin{tabular}{|c|c|c|c|c|c|c|c|c|c|c|}
\hline Caso & Agentes Involucrados & Causas (Factores de Riesgo) & Evento & $\begin{array}{c}\text { Consecuencias o } \\
\text { Impactos }\end{array}$ & $\begin{array}{c}\text { Objetivo(s) } \\
\text { (Estratégico(s)) }\end{array}$ & $\begin{array}{c}\text { Acciones para el } \\
\text { logro del Objetivo }\end{array}$ & \begin{tabular}{|c|}
$\begin{array}{c}\text { Decisiones que se } \\
\text { tomaron }\end{array}$ \\
\end{tabular} & Riesgo & Amenazas & Vulnerabilidades \\
\hline 1 & $\begin{array}{l}\text { Jugador de fútbol } \\
\text { Sus compañeros de juego } \\
\text { Hinchas }\end{array}$ & $\begin{array}{l}\text { (1) No estar en zona segura. } \\
\text { (2) No tener control sobre los } \\
\text { alimentos y bebidas que se } \\
\text { ingiere. } \\
\text { (3) Salir sorteado para prueba } \\
\text { "antidoping". }\end{array}$ & $\begin{array}{c}\text { Examen positivo } \\
\text { por consumir } \\
\text { bebida } \\
\text { contaminada. }\end{array}$ & $\begin{array}{l}\text { (1) Ser sancionado. } \\
\text { (2) Gastar para } \\
\text { defenderse. } \\
\text { (3) Daño en la } \\
\text { reputación. } \\
\text { (4) Perder ingresos } \\
\text { por no poder jugar. }\end{array}$ & $\begin{array}{l}\text { (1) Jugar el Mundial. } \\
\text { (2) Poder trabajar } \\
\text { (jugar). }\end{array}$ & \begin{tabular}{|l|} 
(1) Evitar consumir \\
alimentos en zonas no \\
seguras, dentro de \\
una concentración. \\
(2) Cuidar de no \\
ingerir alimentos no \\
provistos por \\
responsables de \\
concentración. \\
(3) Verificar que todo \\
alimento que se \\
consume esté en zona \\
segura. \\
\end{tabular} & $\begin{array}{l}\text { (1) Consumir } \\
\text { bebida, asumiendo } \\
\text { que se estaba en } \\
\text { zona segura. }\end{array}$ & $\begin{array}{l}\text { No poder trabajar, ni } \\
\text { jugar el mundial por } \\
\text { recibir una sancion } \\
\text { debido a que el } \\
\text { examen "antidoping" } \\
\text { salga positivo al } \\
\text { haber consumido un } \\
\text { alimento } \\
\text { contaminado. }\end{array}$ & $\begin{array}{l}\text { (1) Alimentos } \\
\text { contaminados } \\
\text { en } \\
\text { concentración } \\
\text { fuera de zona de } \\
\text { seguridad. } \\
\text { (2) Recibir } \\
\text { amigos y } \\
\text { familiares en } \\
\text { zonas no } \\
\text { seguras. }\end{array}$ & $\begin{array}{l}\text { (1) No poder } \\
\text { verificar y controlar } \\
\text { todo lo que se } \\
\text { ingiere, incluyendo } \\
\text { aquellos alimentos } \\
\text { que están en la } \\
\text { zona segura. }\end{array}$ \\
\hline 2 & \begin{tabular}{|l|} 
Aereolinea (Aeroperu) \\
Tripulación del Avión \\
Pasajeros y su familiares
\end{tabular} & $\begin{array}{l}\text { (1) Falla del proceso de } \\
\text { lavado del avión (revisiones } \\
\text { de supervisores). } \\
\text { (2) Error humano (olvidar } \\
\text { retirar "masking tape" de } \\
\text { pitots). } \\
\text { (3) Error humano (tripulación } \\
\text { no efectúo revisión). } \\
\text { (4) Externo; era de noche, } \\
\text { nula visibilidad. } \\
\text { (5) Posiblemente apuro para } \\
\text { salir. } \\
\end{array}$ & $\begin{array}{l}\text { Caída de avión en } \\
\text { el mar. }\end{array}$ & \begin{tabular}{|l} 
(1) Muerte de \\
pasajeros y \\
tripulación. \\
(2) Pérdida del avión. \\
(3) Problemas \\
financieros para la \\
aereolinea. \\
(4) El avión no llegó \\
a su destino (no se \\
completo el \\
proceso).
\end{tabular} & $\begin{array}{l}\text { (1) Proteger la vida de } \\
\text { os pasajeros y } \\
\text { tripulación. } \\
\text { (2) Llegar a destino, en } \\
\text { horario establecido. } \\
\text { (3) Proteger la } \\
\text { reputación y patrimonio } \\
\text { de la aereolínea. }\end{array}$ & $\begin{array}{l}\text { (1) Ser cuidadoso de } \\
\text { los procedimientos* } \\
\text { de limpieza del avión. } \\
\text { (2) Tripulación debe } \\
\text { aplicar } \\
\text { procedimientos* para } \\
\text { asegurar vuelo. } \\
\text { * Procedimientos } \\
\text { muestran las acciones } \\
\text { de un proceso }\end{array}$ & \begin{tabular}{|l|} 
(1) Iniciar vuelo, \\
sin haber \\
efectuado \\
verificaciones que \\
proceso de \\
limpieza se haya \\
completado \\
correctamente (se \\
asumió qe todo \\
estaba en orden).
\end{tabular} & \begin{tabular}{|l} 
No llegar al destino, y \\
tripulación y \\
pasajeros pierdan la \\
vida por accidente \\
producido en vuelo, \\
debido a negligencia \\
operativa del personal \\
de limpieza y de la \\
tripulación.
\end{tabular} & 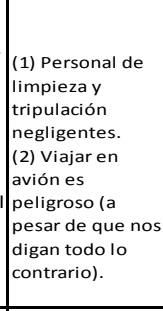 & $\begin{array}{l}\text { (1) Negligencia de } \\
\text { todos los } \\
\text { directamente } \\
\text { involucrados. } \\
\text { (2) Personas no } \\
\text { pueden protegerse } \\
\text { ante la caida de un } \\
\text { avión. }\end{array}$ \\
\hline 3 & \begin{tabular}{|l|} 
Elon Musk \\
Inversionitas que invierten \\
en los titulos de deuda y \\
de capital emitidos por \\
empresas de Musk
\end{tabular} & \begin{tabular}{|l|} 
(1) Productos innovadores \\
prometidos no lleguen al \\
mercado en momento \\
ooportuno. \\
(2) Productos no tengan las \\
(caracteristicas prometidas. \\
(3) Aparezca competencia o \\
la competencia produzzca \\
productos mucho más \\
innovadores. \\
(4) Consumidores no quieran \\
los productos o simplemente \\
no haya consumidores.
\end{tabular} & $\begin{array}{c}\text { Innovaciones y } \\
\text { productos de las } \\
\text { empresas de } \\
\text { Musk no sean } \\
\text { aceptados por el } \\
\text { mercado. }\end{array}$ & $\begin{array}{l}\text { (1) Problemas para } \\
\text { financiar nuevas } \\
\text { innovaciones. } \\
\text { (2) Caída en la } \\
\text { reputación de ser } \\
\text { siempre exitoso. }\end{array}$ & $\begin{array}{l}\text { (1) Ofrecer productos } \\
\text { innovadores a la } \\
\text { sociedad. } \\
\text { (2) Incrementar riqueza } \\
\text { financiera. }\end{array}$ & $\begin{array}{l}\text { (1) Invertir en activos } \\
\text { fisicos y tecnología, y } \\
\text { recursos humanos, } \\
\text { para innovar } \\
\text { productos. } \\
\text { (2) Conseguir capital } \\
\text { de riesgo en los } \\
\text { mercados financieros. }\end{array}$ & \begin{tabular}{|l|} 
1) Musk siempre \\
está enfocado y \\
preparado para \\
entender y \\
gestionar la \\
complejidad. \\
(2) Tomar \\
decisiones de alto \\
riesgo porque se \\
tiene alta \\
tolerancia a la \\
pérdida.
\end{tabular} & $\begin{array}{l}\text { Afrontar alta pérdida } \\
\text { financiera (quiebra } \\
\text { incluida) por haber } \\
\text { invertido en empresas } \\
\text { innovadoras de Elon } \\
\text { Musk. }\end{array}$ & $\begin{array}{l}\text { (1) Poca } \\
\text { inclinación a la } \\
\text { diversificación, } \\
\text { tanto de Elon } \\
\text { Musk como de } \\
\text { sus empresas. }\end{array}$ & $\begin{array}{l}\text { (1) Exceso de } \\
\text { confianza de Elon } \\
\text { Musk }\end{array}$ \\
\hline 4 & \begin{tabular}{|l|} 
Presidente Truman \\
El Estado Norteamericano \\
Los habitantes del planeta
\end{tabular} & \begin{tabular}{|l} 
(1) Escalamiento de conflictos \\
en Corea de Norte por parte \\
de ejercito de Mao Tse Tung. \\
(2) Deseo de poder por parte \\
del General Mac Arthur. \\
(3) Presión por parte de \\
Medios de Comunicación y \\
peblación para "vencer \\
rápidamente la guerra fria". \\
(4) Información asimétrica \\
para obtener información \\
respecto a cómo está armado \\
enememigo.
\end{tabular} & $\begin{array}{l}\text { Bombardeo de } \\
\text { China con } \\
\text { Bombas } \\
\text { Atómicas. }\end{array}$ & $\begin{array}{l}\text { (1) Inicio de la } \\
\text { Tercera Guerra } \\
\text { Mundial. } \\
\text { (2) Posible } \\
\text { destrucción del } \\
\text { planeta. }\end{array}$ & $\begin{array}{l}\text { (1) Evitar la ocurrencia } \\
\text { de un conflicto con } \\
\text { consecuencias } \\
\text { impredecibles ("Tercera } \\
\text { Guerra Mundial"). } \\
\text { (2) Proteger los } \\
\text { intereses del Estado } \\
\text { Norteamericano. } \\
\text { (3) "Ganar" la Guerra } \\
\text { Fria. }\end{array}$ & \begin{tabular}{|l|} 
(1) Actuar y tomar \\
decisiones \\
estratégicas con \\
información \\
verificable y confiable. \\
(2) Contar con \\
personal confiable. \\
(3) Medir la fuerza de \\
los potenciales \\
enemigos. \\
(4) Utilizar la fuerza e \\
información de los \\
aliados.
\end{tabular} & $\begin{array}{l}\text { (1) Actuar con } \\
\text { prudencia. } \\
\text { (2) Dehacerse del } \\
\text { peligro o amenaza } \\
\text { (destitución de } \\
\text { Mac Arthur). } \\
\text { (3) Evitar y hasta } \\
\text { ignorar la presión } \\
\text { de la opinión } \\
\text { pública. }\end{array}$ & $\begin{array}{l}\text { Se inicie un conflicto } \\
\text { con consecuencias } \\
\text { impredecibles por el } \\
\text { uso de bombas } \\
\text { atomicas para } \\
\text { contener el avance } \\
\text { del Ejercito Chino en } \\
\text { Corea del Norte. }\end{array}$ & $\begin{array}{l}\text { (1) General } \\
\text { MacAtrtur } \\
\text { (2) Mao Tse } \\
\text { Tung ysu } \\
\text { ejercito. } \\
\text { (3) Opinión } \\
\text { Pública }\end{array}$ & $\begin{array}{l}\text { (1) No saber la } \\
\text { capacidad y fuerza } \\
\text { del enemigo directo } \\
\text { y de sus aliados. } \\
\text { (2) Truman no era } \\
\text { un buen } \\
\text { comunicador. } \\
\text { (3) Truman no } \\
\text { contaba con la } \\
\text { simpatia de los } \\
\text { medios y de la } \\
\text { población. }\end{array}$ \\
\hline
\end{tabular}

Fuente: Elaboración propia 


\section{REFERENCIAS}

[1] Bartlett, C. (2013). Air crashes and miracle landings: sixty narratives (How, When... And Most Importantly Why). London, UK: OpenHatch Books.

[2] Bernstein, P. (1996). Against the gods:the remarkable story of risk. New York: John Wiley \& Sons, Inc.

[3] Brands, H. (2016). The general vs. the president: Mac Arthur and Truman at the brink of nuclear war. New York: Anchor Books.

[4] C. Spetzler, H. W. (2016). Decision quality: value creation from better decisions. Hoboken, New Jersey: John Wiley \& Sons, Inc.

[5] Freedman, L. (2013). Strategy: a history. Oxford: Oxford University Press.

[6] Hnyilicza, E. (2005). El abrazo invisible: hacia las reformas de tercera generación. Lima, Perú: Fondo Editorial de la Universidad San Martin de Porras.

[7] Hubbard, D. (2009). The failure of risk management: Why it's broken and how to fix it. Hoboken, New Jersey: John Wiley \& Sons, Inc.

[8] International Organization for Standardization (Febrero de 2018). ISO 31000:2018 Risk management Guidelines (Second Edition)
[9] Merton, Z. B. (1999). Finanzas. México: Prentice Hall.

[10] Purdy, G. (Mayo de 2018). Controversial thoughts about modern day risk management in non-financial companies: The most amazing risk management interview...ever...Alex Sidorenko talks to Grant Purdy on why most risk management practices don't work. Risk Academy Blog. (A. Sidorenko, Entrevistador)

[11] Ripley, A. (2008). The unthinkable: who survives when disaster strikes and why. New York: Three Rivers Press.

[12] Taleb, N. (2004). Fooled by randomness: the hiddenn role of chance in life and markets. New Yok: Random House.

[13] Taleb, N. (2010). The black swan: the impact of the highly improbable. New York: Random House.

[14] The Economist. (10 de Febrero de 2018). How Elon Musk does it: The Falcon Heavy's Creator is trying to change more worlds than one-Faliure is most definiively an option. The Economist. Recuperado de https:// www.economist.com/briefing/2018/02/10/ the-falcon-heavys-creator-is-trying-tochange-more-worlds-than-one

[15] Vance, A. (2015). Elon Musk: Tesla, space $X$ and the quest for a fantastic future. New York: Harper Collins. 